\title{
New Hope for Alzheimer's Disease
}

\author{
B. Vellas ${ }^{1}$, P. Aisen ${ }^{2}$ \\ 1. Gerontopole and Alzheimer's Disease Research and Clinical Center, Toulouse University Hospital, France; 2. USC ATRI, San Diego, CA USA \\ Corresponding Author: B. Vellas, Gerontopole and Alzheimer's Disease Research and Clinical Center, Toulouse University Hospital, France, vellas.b@chu-toulouse.fr
}

O ur field has reached a time of excited anticipation. After many years of frustration, we can now expect dramatic change for the better.

\section{Diagnosis and tracking progression with new blood biomarkers for Alzheimer's}

Plasma assays that reflect Alzheimer's pathology in brain have now been validated across many studies (1-3) representing an astounding advance in the field. Mass spectrometry-based plasma $A \beta 42$ / A $\beta 40$ assays are highly correlated to cerebrospinal fluid assyas and amyloid PET SUVr measures. These blood tests are now being used as pre-screening tests prior to PET scanning to greatly reduce the burden and costs associated with scanning for trial recruitment. It is plausible that plasma assays may even obviate the need for PET scanning. Measurement of phosphotau species such as ptau217 (2) in plasma are tightly correlated to both amyloid and tau pathology and can likewise be used for screening and longitudinal follow-up in trials, potentially indicating downstream effects of candidate therapies targeting amyloid or tau. Preliminary evidence suggest that plasma amyloid and tau assays may allow identification of individuals on the Alzheimer's disease spectrum even before PET scans become abnormal. The impact of this new technology on AD disease-modifying drug development will be profound. Further, we can foresee the possibility that plasma biomarkers may prove useful in establishing risk prior to any measurable brain pathology, facilitating the advance of primary prevention trials for Alzheimer's.

\section{New hope for effective disease-modifying therapies}

After decades of disappointments, a candidate diseasemodifier, aducanumab, is now under review at the FDA (4). A second amyloid-reducing antibody, donanemab, has shown positive clinical results in a Phase 2 trial (5), and two other antibodies in this class, gantenerumab and lecanemab, also show encouraging results. A number of factors have contributed to this progress, including successful management of the adverse effects of amyloid removal (amyloid-related imaging abnormalities, or
ARIA), higher dose therapy and selection of pre-dementia participants likely to show clinical benefit (4). New studies are in progress or planning to bring anti-amyloid antibodies into still earlier stages of disease, presumably further increasing the possible clinical benefit. The AntiAmyloid Treatment in Asymptomatic Alzheimer's (A4) trial, treating individuals at the asymptomatic stage of disease with solanezumab, an antibody targeting monomeric amyloid, will be completed by the beginning of 2023. The AHEAD platform includes two trials: a phase 3 trial of lecanemab in asymptomatic Alzheimer's (A45) and a phase 2 trial of lecanemab in individuals with intermediate (sub-threshold) levels of amyloid. We are optimistic that multiple therapies, initially targeting amyloid but eventually tau and other targets as well, will reach the clinic in the coming years. Secondary and even primary prevention of Alzheimer's may follow.

\section{New hope for clinical practice}

The World Health Organization (WHO) Global strategy on ageing and health provides a policy framework to align health systems to the needs of older populations $(6,7)$. ICOPE program promotes person-centered care on older peoples' intrinsic capacity and ability to function where they live. ICOPE include cognition as part of the major intrinsic capacities to be maintained for an healthy aging and will encourage primary care professional to assess and monitor memory. ICOPE digital monitoring (8) associated to blood biomarkers will make A.D early diagnosis available to all.

Remarkable developments in geriatric assessment and Alzheimer's therapeutic research herald major advances that will improve the lives of millions of aging individuals. Continued collaboration among academic scientists, clinicians, advocacy groups, philanthropists, diverse communities and the biotech and pharmaceutical industries will assure continued progress toward a bright future.

Conflict of interest: Dr. Aisen reports grants from Janssen, NIA, FNIH, Alzheimer's Association, and Eisai, personal fees from Merk, Biogen, Roche, ImmunoBrain Checkpoint, Abbvie, Rainbow Medical, and personal fees from Shionogi, outside the submitted work; Dr. Vellas reports grants from Lilly, Merck, Roche, Lundbeck, Biogen, grants from Alzheimer's Association, European Commission, personal fees from Lilly, Merck, Roche, outside the submitted work. 


\section{References}

1. Schindler SE, Bollinger JG, Ovod V, Mawuenyega KG, Li Y, Gordon BA, Holtzman DM, Morris JC, Benzinger TLS, Xiong C, Fagan AM, Bateman RJ. High-precision plasma $\beta$-amyloid $42 / 40$ predicts current and future brain amyloidosis. Neurology. 2019 Oct 22;93(17):e1647-e1659. doi: 10.1212/ WNL.0000000000008081. Epub 2019 Aug 1. PMID: 31371569; PMCID: PMC6946467.

2. Janelidze S, Berron D, Smith R, Strandberg O, Proctor NK, Dage JL, Stomrud E, Palmqvist S, Mattsson-Carlgren N, Hansson O. Associations of Plasma Phospho-Tau217 Levels With Tau Positron Emission Tomography in Early Alzheimer Disease. JAMA Neurol. 2021 Feb 1;78(2):149-156. doi: 10.1001/ jamaneurol.2020.4201. PMID: 33165506; PMCID: PMC7653537.

3. Karikari TK, Benedet AL, Ashton NJ, Lantero Rodriguez J, Snellman A, SuárezCalvet M, Saha-Chaudhuri P, Lussier F, Kvartsberg H, Rial AM, Pascoal TA Andreasson U, Schöll M, Weiner MW, Rosa-Neto P, Trojanowski JQ, Shaw LM, Blennow K, Zetterberg H; Alzheimer's Disease Neuroimaging Initiative. Diagnostic performance and prediction of clinical progression of plasma phospho-tau181 in the Alzheimer's Disease Neuroimaging Initiative. Mol Psychiatry. 2021 Feb;26(2):429-442. doi: 10.1038/s41380-020-00923-z. Epub 2020 Oct 26. PMID: 33106600
4. Aisen PS, Cummings J, Doody R, Kramer L, Salloway S, Selkoe DJ, Sims J, Sperling RA, Vellas B. The Future of Anti-Amyloid Trials. J Prev Alzheimers Dis. 2020;7(3):146-151. doi: 10.14283/jpad.2020.24. PMID: 32463066.

5. Mintum M.A, Lo A.C, Evans C.D et al Donanemap in Early Alzheimer's disease. N Engl J Med 2021; 384:1691-1704. DOI: 10.1056/ NEJMoa2100708

6. Integrated care for older people Guidelines on community-level interventions to manage declines in intrinsic capacity WHO.2017; (www.who.int/ageing/ health-systems /icope, Accessed 24 February 2019).

7. Banerjee A, Sadana R. Editorial: Integrated Care for Older People (ICOPE): From Guidelines to Demonstrating Feasibility. J Frailty Aging. 2021;10(2):84-85. doi: 10.14283/jfa.2020.40. PMID: 33575695

8. Piau A, Steinmeyer Z, Cesari M, Kornfeld J, Beattie Z, Kaye J, Vellas B, Nourhashemi F. Intrinsic Capacitiy Monitoring by Digital Biomarkers in Integrated Care for Older People (ICOPE). J Frailty Aging. 2021;10(2):132-138. doi: $10.14283 /$ jfa.2020.51. PMID: 33575701 